\title{
Comparative Evaluation of Short Fiber-reinforced Composite Resin Thickness on Fracture Resistance of Class II Composite Restoration: An In Vitro Study
}

\author{
Calvina Hartanto ${ }^{1}$, Wandania Farahanny ${ }^{2}$, Dennis Dennis ${ }^{3}$
}

\begin{abstract}
Aim: This study aims to evaluate the difference in fracture resistance of the short fiber-reinforced composite (SFRC) thickness as intermediate layer of class II composite restoration.

Materials and methods: Thirty human maxillary premolars were selected and divided into three groups. In groups I, II, and III, class II cavities were prepared. Groups I and II were restored with $2 \mathrm{~mm}$ and $4 \mathrm{~mm}$ thickness of SRFC as intermediate layer. Group III as control group was restored with nanohybrid composite. Thermocycling procedure was performed manually 250 times. Fracture resistance was measured by using Universal Testing Machine. Data were analyzed statistically using One-way Analysis of Variance (ANOVA) and post hoc least significant difference (LSD) test. Results: The result showed that group II had the highest fracture resistance and group III had the lowest fracture resistance. The difference between groups was statistically significant ( $p$ value $<0.05)$.

Conclusion: The conclusion showed that adding $4 \mathrm{~mm}$ of SFRC as an intermediate layer increased the fracture resistance.

Clinical significance: The use of SFRC as intermediate layer enhanced the fracture resistance of class II composite restoration.

Keywords: Class II, Fracture resistance, Nanohybrid composite, Short fiber-reinforced composite.

The Journal of Contemporary Dental Practice (2020): 10.5005/jp-journals-10024-2965
\end{abstract}

\section{INTRODUCTION}

Missing of tooth structure often occurs due to trauma, caries, and cavity preparation. Restoration is needed to replace the missing tooth structure. An ideal restoration in a tooth should be able to maintain the esthetics and function, preserve the remaining tooth structure, and prevent microleakage. One of the objectives of tooth restoration is to reestablish its fracture resistance when subjected to occlusal load. In final restorations, composite resin shows an increased resistance to fracture, as it replaces biological tissue esthetically and functionally. ${ }^{1-3}$

A successful restoration is determined by its longevity in the oral cavity, and other factors, such as cavity design, size of cavity, type of teeth, and restoration material. Based on the prospective studies for around 10 years, the survival proportion of Class II restorations ranged from 58.3 to $100 \%$, and the retrospective studies varied from 55.1 to $89.7 \% .{ }^{4,5}$ The prevailing reasons for restoration failure are fracture and secondary caries. Fracture is a considerable risk to posterior restorations, regardless of the lifespan or the age of these restorations. ${ }^{6}$

In class II cavity, there is loss of the proximal and marginal ridge. This causes the tooth stiffness to reduce significantly by 2.5 -fold, resulting in an overall $46 \%$ reduction of tooth stiffness. Therefore, restoration material with high fracture resistance is highly recommended in cases where it is subjected to heavy load as in cases of class II.

The usage of intermediate layer preceding the placement of packable composite resin may play an important role in the long-term success of posterior direct composite restorations. ${ }^{8}$ The frequently used intermediate layer is flowable composite. $A$ research by Al-lbrahemi states that the increased thickness of flowable composite placed as intermediate layer can increase

\begin{abstract}
${ }^{1-3}$ Department of Conservative Dentistry, Faculty of Dentistry, Universitas Sumatera Utara, Medan, Sumatera Utara, Indonesia

Corresponding Author: Wandania Farahanny, Department of Conservative Dentistry, Faculty of Dentistry, Universitas Sumatera Utara, Medan, Sumatera Utara, Indonesia, Phone: +6282160665872, e-mail: wandania@usu.ac.id

How to cite this article: Hartanto C, Farahanny W, Dennis D. Comparative Evaluation of Short Fiber-reinforced Composite Resin Thickness on Fracture Resistance of Class II Composite Restoration: An In Vitro Study. J Contemp Dent Pract 2020;21(11):1201-1204.

Source of support: Nil

Conflict of interest: None

the compressive strength. ${ }^{9}$ However, flowable composites have lower mechanical and physical properties compared to standard restorative composites and are not recommended for usage in stress-bearing areas. ${ }^{8}$

The durability of a dental restoration is affected by its mechanical properties. The lost dental tissues, caused by caries or endodontic treatment of anterior or posterior tooth, should be replaced with a potential restorative material which have adequate strength and retention to protect the tooth against masticatory forces and preserve the remaining structure. Considering the compressive masticatory forces in the posterior region, the material should endure these forces. Compressive strength is one of the most important mechanical properties of posterior restorative material. A material with higher or lower amount of property will adversely affect on longevity of the tooth structure and the restoration. A restoration with lower compressive strength than the natural tooth, tends to fail or fracture and might lead to periodontal problems or extraction of the tooth. ${ }^{9}$
\end{abstract}


The drawback of using conventional composites were stress development due to polymerization shrinkage and insufficient fracture resistance. These disadvantages encouraged the researchers to search for a new generation of composite that has better fracture strength with less polymerization shrinkage. ${ }^{2} \mathrm{~A}$ material with high fracture resistance has the ability to better prevent crack initiation and propagation. ${ }^{10}$ The strength of the materials depends on their compositions, such as filler. ${ }^{11}$ Research by Belli said that application of a fiber layer in a restorative material might increase the load-bearing capacity of the restoration and could prevent crack propagation from the restoration to the tooth. ${ }^{12}$

In 2013, short fiber-reinforced composite resin (SFRC) was introduced to the market with the goal to substitute the missing dentin with a material having a similar behavior. SFRC has clinically shown to mimic the stress-absorbing properties of dentin. SFRC is proposed to be used as bulk base in high stress-bearing areas for restoring vital and non-vital teeth. The fiber reinforcement is partially to strengthen the structurally compromised tooth internally and to prevent the occurrence of fractures. ${ }^{6}$ The SFRC has also revealed control of the polymerization shrinkage stress by fiber orientation; thus, marginal microleakage was reduced compared with conventional particulate filler composite resins. By using a SFRC composite substructure under conventional composite resin, the static load-bearing capacity of this combination could be improved. ${ }^{13}$

Due to lack of substantial conclusive literature on the strength and success of this material, this in vitro study was designed to compare the fracture resistance of short fiber-reinforced composite thickness as intermediate layer of class II composite restoration.

\section{Materials and Methods}

This research constituted a laboratory experimental study using post test only control group design. Thirty intact and caries-free human maxillary premolars extracted for orthodontic reasons were used and approved by Health Research Ethics Committee, Medical Faculty of Universitas Sumatera Utara. All participants gave out informed consents prior to collecting the teeth samples. Gross cleaning was done by scaler and stored in saline solution. Thirty premolars were divided into three groups with 10 samples in each group. All samples were prepared with class II box-only cavity with mesiodistal length $2 \mathrm{~mm}$, buccopalatal width $4 \mathrm{~mm}$, verified by using a caliper, and depth $5 \mathrm{~mm}$ measured using a periodontal probe.

All of the samples were randomly divided into three groups according to the thickness of SFRC as intermediate layer:

Group I: Class II cavity, restored with $2 \mathrm{~mm}$ thickness of SFRC (EverX Posterior, GC, Japan) as intermediate layer and $3 \mathrm{~mm}$ of nanohybrid composite (Filtek ${ }^{\mathrm{TM}}$ Z250XT, 3M ESPE, USA).

Group II: Class II cavity, restored with $4 \mathrm{~mm}$ thickness of SFRC (EverX Posterior, GC, Japan) as intermediate layer and $1 \mathrm{~mm}$ of nanohybrid composite (Filtek ${ }^{\text {TM }}$ Z250XT, 3M ESPE, USA).

Group III: Class II cavity, restored with nanohybrid composite (Filtek ${ }^{\text {TM }}$ Z250XT, 3M ESPE, USA).

The cavity surfaces were etched with $37 \%$ phosphoric acid for 15 seconds and rinsed with water for 15 seconds. The universal bonding agent (G-Premio Bond, GC, Japan) was applied to the cavity walls with a microapplicator and light cured for 20 seconds. SFRC was applied with 2- and 4-mm thickness with bulk technique and measured with a periodontal probe. The final layer of nanohybrid composite was applied with an incremental technique, and each layer was light-cured for 20 seconds. A fine finishing bur and spiral polishing disk were used to polish all samples.

The teeth from all the groups were stored in artificial saliva solution for 24 hours. Thermocycling procedure was performed manually 250 times, with soaking the samples in iced water $\left(5^{\circ} \mathrm{C}\right)$ and water bath of $55^{\circ} \mathrm{C}$ for 30 seconds and dwell time of 10 seconds.

The root surfaces were covered with molten baseplate wax to simulate the periodontal ligament. All the samples were embedded and stabilized in a self-cure acrylic resin cylinder until $1 \mathrm{~mm}$ below cementoenamel junction. A special acrylic mold was prepared to provide the long axis of the teeth at a $13.5^{\circ}$ angle to the vertical plane. Samples were positioned in the mold and held at a $13.5^{\circ}$ angle for loading. All samples were subjected to fracture resistance testing at a crosshead speed of $1 \mathrm{~mm} /$ minute using Servopulser Universal Testing Machine (Fig. 1). The fracture load was recorded, and the data obtained were subjected to statistical analysis by 1-way analysis of variance (ANOVA) and post hoc least significant difference (LSD) test ( $p$ value $<0.05$ ).

\section{Results}

Groups were compared using one-way ANOVA. The descriptive statistics that represent the mean, standard deviation, and $p$ value are shown in Table 1. The mean value is presented in Figure 2. Group II, which used $4 \mathrm{~mm}$ SFRC thickness, showed higher fracture resistance (535.71 $\mathrm{N} \pm 74.03)$ than group I (444.21 $\mathrm{N} \pm 82.79)$ and group III (438.65 $\mathrm{N} \pm 107.75)$.

In this study, the result of fracture resistance with statistical analysis indicated that there was a significant difference between groups ( $p$ value $<0.05$ ). Post hoc LSD test was performed for multiple comparisons between groups (Table 2). It was observed that group II (4 mm SFRC thickness) showed a significant difference when compared with group I ( $2 \mathrm{~mm}$ SFRC thickness) and group III (nanohybrid composite) ( $p$ value $<0.05$ ). Group I $(2 \mathrm{~mm}$ SFRC thickness) showed no significant difference in fracture resistance when compared with group III (nanohybrid composite) ( $p$ value $>0.05$ ). The pairwise comparison of fracture resistance between groups showed that the fracture load recorded for $4 \mathrm{~mm}$ SFRC thickness group was significantly higher than those group for $2 \mathrm{~mm}$ SFRC thickness and nanohybrid composite group ( $p$ value $<0.05$ ). The null hypothesis that there were no significant differences of

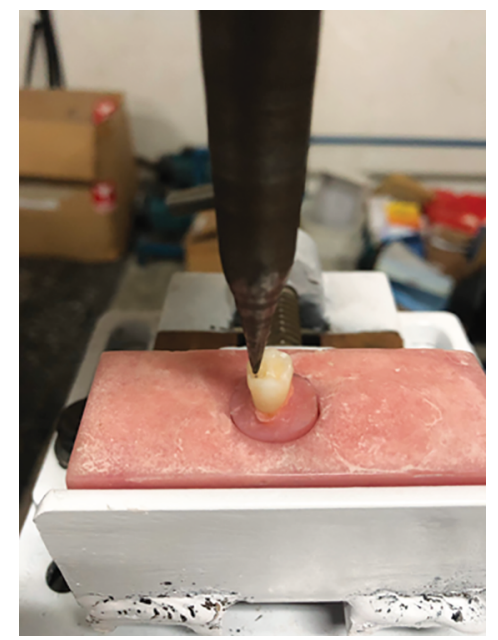

Fig. 1: Fracture test using universal testing machine 
Comparative Evaluation of SFRC Thickness on Fracture Resistance

Table 1: Descriptive data of three different groups fracture resistance using one-way analysis of variance

\begin{tabular}{|c|c|c|c|c|c|c|c|c|}
\hline & \multirow[b]{2}{*}{$N$} & \multirow[b]{2}{*}{ Mean } & \multirow[b]{2}{*}{ Std. deviation } & \multirow[b]{2}{*}{ Std. error } & \multicolumn{2}{|c|}{ 95\% confidence interval for mean } & \multirow[b]{2}{*}{ Minimum } & \multirow[b]{2}{*}{ Maximum } \\
\hline & & & & & Lower bound & Upper bound & & \\
\hline Group I & 10 & 444.21440 & 82.966128 & 26.236193 & 384.86401 & 503.56479 & 279.692 & 581.140 \\
\hline Group II & 10 & 535.70720 & 74.025308 & 23.408858 & 482.75268 & 588.66172 & 401.408 & 659.344 \\
\hline Group III & 10 & 438.64800 & 107.748008 & 34.072912 & 361.56972 & 515.72628 & 252.448 & 566.734 \\
\hline Total & 30 & 472.85653 & 97.408284 & 17.784238 & 436.48368 & 509.22938 & 252.448 & 659.344 \\
\hline
\end{tabular}

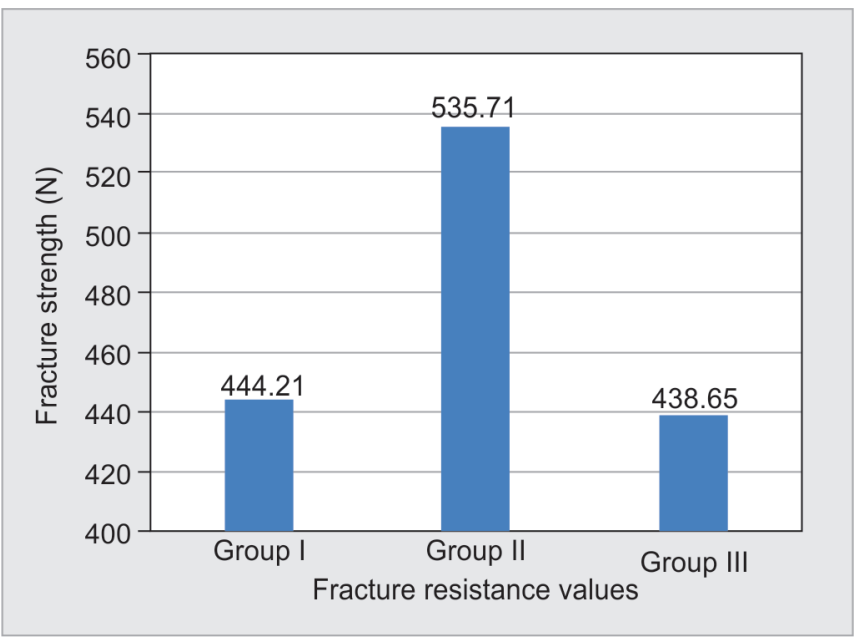

Fig. 2: Means value of fracture resistance of all groups (Newton)

Table 2: Post hoc least significant difference test result shows the mean difference and $p$ value of the fracture resistance between groups

\begin{tabular}{lll}
\hline Variable & Mean difference & p value \\
\hline Group I-Group II & 91.49 & 0.03 \\
Group I-Group III & 5.57 & 0.89 \\
Group II-Group III & 97.06 & 0.02 \\
\hline
\end{tabular}

fracture resistance among the SFRC thickness as intermediate layer was rejected because there were statistically significant differences.

\section{Discussion}

When subjected to occlusal loads, a tooth restoration should restore the natural tooth's fracture resistance. ${ }^{2}$ Considering the compressive masticatory forces in the posterior region, the material should endure these forces. ${ }^{9}$

There are two methods currently used for assessing the fracture resistance of dental restoration: the compressive static fracture test and dynamic fracture test. The compressive static fracture test was performed using the Universal Testing Machine. Although an attempt was made to simulate the oral environment, the conditions were not identical to those of the intraoral. The applied load was only in one direction and one point, which did not simulate many ways of masticatory force exertion. A static test is often regarded as clinically less relevant, since such abrupt loading never occurs. However, dynamic fracture testing is claimed to better predict the clinical situation and has been considered as the standard to assess or predict the influence of mouth-motion fatigue on dental restoration. ${ }^{14,15}$

In the compressive test, the loading angulation is paramount. In this study, the load was directed to the area of the restoration at a $13.5^{\circ}$ angle because $13.5^{\circ}$ angle is the typical loading angle used in dental applications. The load at failure decreases as the loading angulation increases. ${ }^{16}$

The present study compared the thickness of SFRC in terms of fracture resistance. Based on the results, the highest mean fracture resistance was seen in the 4-mm SFRC thickness group, followed by 2-mm SFRC thickness, and nanohybrid composite group has the lowest mean of fracture resistance. This result agrees with previous studies, and Al-lbrahemic observed that the fracture resistance increases as the flowable composite thickness increases. The fracture resistance of a restoration may be due to filler loading and mechanical properties of the material. Increasing the filler loading will increase the fracture resistance. ${ }^{9}$

In this study, the composite consisted of short fibers, whereas in the aforementioned research, the intermediate layer did not contain an additional component. The function of SFRC is assumed to be based on supporting the superficial conventional composite and behaving as a crack arrest barrier. This bilayered restoration is able to mimic the natural behavior of enamel and dentin. The presence of fibers prevents and stops the crack propagation throughout the restoration. Adequate adhesion among fiber and matrix, which affords good load transfer, assures that the load is transferred to the stronger fiber, and it is the fiber that actually works as reinforcement. Voids that appear among the fiber and the polymer matrix is caused by insufficient adhesion and would act as initial fracture sites in the matrix and facilitate the breakdown of the material. Random fiber orientation and lowered cross-linking density of the polymer matrix by the semi-interpenetrating polymer network (semi-IPN) structure has a significant role in enhancing mechanical properties. Reinforcing effect of the fibers fillers is based not only on stress transfer from polymer matrix to fibers but also depends on behavior of individual fiber that acts as a crack stopper. $2,6,9,10,17$

In relation to the SFRC, Garoushi et al. observed that EverX Posterior had fiber length distribution between 0.3 and $1.5 \mathrm{~mm}$, which is in the range of the reported critical fiber length and desired aspect ratio. The critical fiber lengths of E-glass with bis-GMA polymer matrix vary between 0.5 and $1.6 \mathrm{~mm}$. The stress transfer from polymer matrix to the fibers only possible if the fibers have a length equivalent or greater than the critical fiber length and essential for producing effective reinforcement. The amount, type, size, and load of fiber in the composite also affect its mechanical properties. Additionally, the fibers are silanized and are therefore able to chemically connect to the matrix. As a consequent of these features, the SFRC is able to reinforce the dental structures even in case of extreme loading conditions. Since these fibers show random orientation, they can reduce the polymerization stress generated by the composite resin in all directions. 6,10,18,19

Stress generated during polymerization shrinkage of composites have potential to cause an adhesive failure or microcracking of restorative material and/or at interface with tooth structure. The microcracks can predispose the tooth to fracture and affect the 
capability of the restoration to resist occlusal forces. Therefore, short fiber fillers might absorb some of the polymerization shrinkage stresses and increase the stress-relieving capacity of the matrix, and this could decrease the marginal microleakage and improve the adaptation of the material. ${ }^{20,21}$

Tsujimoto et al. observed that the SEM image of fracture surface of SFRC after fracture resistance measurement indicate the possibility that short E-glass fiber retard crack propagation along the fracture line. If the minor crack propagates through this kind of material, it encounters a fiber and cannot grow further. SFRC can mitigate damage and dissipate energy, which greatly improves their mechanical properties by preventing brittle failure and avoiding the loss structural intergrity. ${ }^{17,18}$

The two essential factors that strengthen the SFRC are millimeter-scale short fibers and semi-IPN structure. The millimeterscale short fibers have an ability to bridge, stretch, divert, and withstand the opening and propagation of the crack, therefore inducing the stress intensity at the crack tip. The stretching capacity of fibers may allow the crack bridging and the crack blunting mechanisms to occur. During crack bridging, discontinuous fibers would probably stretch between the edges of the propagating crack causing blunting of the initially sharp crack. This would probably minimize or avert the crack propagation. The semi-IPN consists of both linear and cross-linked polymer phases. The linear phase can be dissolved if a suitable adhesive resin is added on its surface, thus enabling the reactivation of the material and also true chemical bonding to it. Then, the polymethyl acrylate within the semi-IPN structure which reduces the stiffness of the cross-linked resin monomer must be contributing to the toughening of the material. ${ }^{19,22}$

Increased filler loading generally increases physical and mechanical properties. This determines a restoration's clinical performance and durability. If the thickness of the material increases, the filler amount also increases. In this study, there is no significant difference between groups 1 and 3, means that with the addition of $2 \mathrm{~mm}$ SFRC has the same effect as the control group, where both groups can bear the equal forces. Increasing the SFRC thickness leads to the increase of the fiber content in the filler, resulting in the highest fracture resistance observed in group II. The fibers act as stress absorber and crack stopper, and an increased fiber amount will reduce fracture probability. The limitation of this study is using static load, as this could not mimic the clinical condition. Further studies should be conducted to evaluate the fracture resistance using the dynamic or intermittent load fracture test to simulate the actual masticatory movement, and the finite element analysis study sought to assess the stress distribution at the interface in restoration.

\section{Conclusion}

From the results of the study, it can be concluded that the increase in SFRC thickness as intermediate layer increases the fracture resistance on class II restoration.

\section{References}

1. Heymann HO, Swift Jr EJ, Ritter AV. Sturdevant's Art and Science of Operative Dentistry. 6th ed., USA: Mosby Co; 2012. p. 429.

2. Kumar A, Tekriwal S, Rajkumar B, et al. A comparative evaluation of fracture resistance of fiber reinforced composite, flowable composite, and a core build up material: an in-vitro study. Int J Curr Res 2016;8(6):32379-32380.

3. Cramer NB, Stansbury JW, Bowman CN. Recent advances and developments in composite dental restorative material. J Dent Res 2011;90(4):402. DOI: 10.1177/0022034510381263.

4. Demarco FF, Collares K, Correa MB, et al. Should my composite restorations last forever? why are they failing? Braz Oral Res 2017;31(suppl 1):92-93. DOI: 10.1590/1807-3107bor-2017.vol31.0056.

5. Kubo S. Longevity of resin composite restorations. Japan Dent Sci Rev 2011:47(1):43-55. DOI: 10.1016/j.jdsr.2010.05.002.

6. Garoushi S, Gargoum A, Vallittu PK, et al. Short fiber-reinforced composite restorations: a review of the current literature. J Invest Clin Dent 2018;1(1):1-3. DOI: 10.1111/jicd.12330.

7. Chowdhury D, Guha C, Desai P. Comparative evaluation of fracture resistance of dental amalgam, Z350 composite resin and Cention-N restoration in class II cavity. IOSR-JDMS 2018;17(4):52.

8. Kasraei S, Azarsina M, Majidi S. In vitro comparison of microleakage of posterior resin composites with and without liner using two-step etch-and-rinse and self-etch dentin adhesive systems. Oper Dent 2011;36(2):214. DOI: 10.2341/10-215-L.

9. Al-Ibrahemi JF, Mohammed R. The influence of different thickness of flowable composite base materials on compressive strength of composite restorations. J Genet Environ Resour Conserv 2015;3(1): 53-58.

10. Garoushi S, Vallittu PK, Lassila L. Mechanical properties and wear of five commercial fiber-reinforced filling materials. Chin J Dent Res 2017;20(3):137-143.

11. Anusavice KJ, Shen C, Rawls HR. Phillips' Science of Dental Material. 12th ed., USA: Elsevier; 2013. p. 280.

12. Belli S, Eskitascioglu G. Biomechanical properties and clinical use of a polyethylene fiber post-core material. Int Dent South Africa 2006;8(3):21.

13. Garoushi S, Mangoush E, Vallittu PK, et al. Short fiber reinforced composite: a new alternative for direct onlay restorations. Open Dent J 2013;7(1):182. DOI: 10.2174/1874210601307010181.

14. Nie EM, Chen $X Y$, Zhang $C Y$, et al. Influence of masticatory fatigue on the fracture resistance of the pulpless teeth restored with quartz-fiber post-core and crown. Int J Oral Sci 2012;4(4):218-220. DOI: 10.1038/ ijos.2012.78.

15. Makramani A, Razak A, Yi NM, et al. Effect of restorative techniques on fracture resistance of endodontically treated premolars. J Stomatol 2013;3(07):379-385. DOI: 10.4236/ojst.2013.37064.

16. Ozgunaltay G, Gorucu J. Fracture resistance of class II packable composite restorations with and without flowable liners. J Oral Rehabil 2005;32(2):111-115. DOI: 10.1111/j.1365-2842.2004.01364.x.

17. Tanner J, Tolvanen M, Garoushi S, et al. Clinical evaluation of fiberreinforced composite restorations in posterior teeth - results of 2.5 year follow-up. Open Dent J 2018;12:482.

18. Tsujimoto A, Barkmeier WW, Takamizawa T, et al. Mechanical properties, volumetric shrinkage and depth of cure of short fiberreinforced resin composite. Dent Mater J 2016;35(3):418-419. DOI: 10.4012/dmj.2015-280.

19. Frater M, Forster A. New generation of short-fiber reinforced composite restorations of the posterior dentition. Int Dent - Afr Edit 2018;9(5):7-8.

20. Garoushi SK, Hatem M, Lassila LVJ, et al. The effect of short fiber composite base on microleakage and load-bearing capacity of posterior restorations. Acta Biomater Odontol Scand 2015;1(1):10-11. DOI: 10.3109/23337931.2015.1017576.

21. Moosavi H, Zeynali M, Pour ZH. Fracture resistance of premolars restored by various types and placement techniques of resin composites. Int J Dent 2012;2012:1-2. DOI: 10.1155/2012/973641.

22. Bijelic-Denova J, Garoushi S, Lasilla LVJ, et al. Mechanical and structural characterization of discontinuous fiber-reinforced dental resin composite. J Dent 2016:5-8. DOI: 10.1016/j.jdent.2016.07.009. 